\title{
Short Approach Towards New Isocoumarins and Dihydroisocoumarins and Investigation of their Cytotoxic Activities
}

\author{
Isolde Wetzel, Franz Bracher, and Jürgen Krauss \\ Department Pharmazie - Zentrum für Pharmaforschung, Ludwig-Maximilians-Universität München, \\ Butenandtstraße 5-13, 81377 München, Germany
}

Reprint requests to Dr. Jürgen Krauss. Fax: +49-89-2180 77171.

E-mail: hjkra@cup.uni-muenchen.de

Z. Naturforsch. 2009, 64b, 313-318; received November 25, 2008

\begin{abstract}
3-Substituted isocoumarins were prepared in a short and efficient way from 2-iodobenzoic acid and terminal alkynes in a one-pot Sonogashira reaction. Catalytic hydrogenation gave the corresponding dihydroisocoumarins. The cytotoxic activities of the resulting compounds against human leukaemia cell line (HL 60) were determined in a MTT assay, and structure-activity relationships are discussed.
\end{abstract}

Key words: Isocoumarin, Dihydroisocoumarin, Sonogashira Reaction, Hydrogenation, Cytotoxic Activity

\section{Introduction}

Isocoumarins are a large and structurally diverse class of bioactive natural products with widespread occurance in living organisms [1], and considerable work has been published over decades about their chemistry [2] and biology [3]. A considerable number of natural and synthetic isocoumarins showed significant cytotoxic and antitumor activity. Among those are dihydroisocoumarins like AI-77-B (A), as well as numerous isocoumarins bearing substituents at $\mathrm{C}-3$ (Fig. 1). The dihydroisocoumarin AI-77-B (A) shows in vitro cytotoxicity against human malignant $\mathrm{A} 375$ S2 and human cervical cancer HeLa cells [4]. The paraphaeosphaerins (B) recently isolated from cultures of Paraphaeosphaeria quadriseptana [5] are biogenetically related to the cytotoxic plant metabolites monocillin I and radicicol. NM-3 (C) is a synthetic analog of cytogenin $(\mathbf{E})$, and potentiates antineoplastic effects of other chemotherapeutic agents and inhibits angiogenesis [6]. This compound is in phase I clinical trials. The isocoumarin 185322 (D) [7], an ana$\log$ of NM-3, is an inhibitor of microtubule assembly, and induces mitotic arrest and apoptosis of multiple myeloma cells.

The biological activities of the abovementioned and other isocoumarins and dihydroisocoumarins $[8,9]$ make this class of compounds interesting leads for development of new anticancer drugs. The most prominent structural features found in the bioactive com-

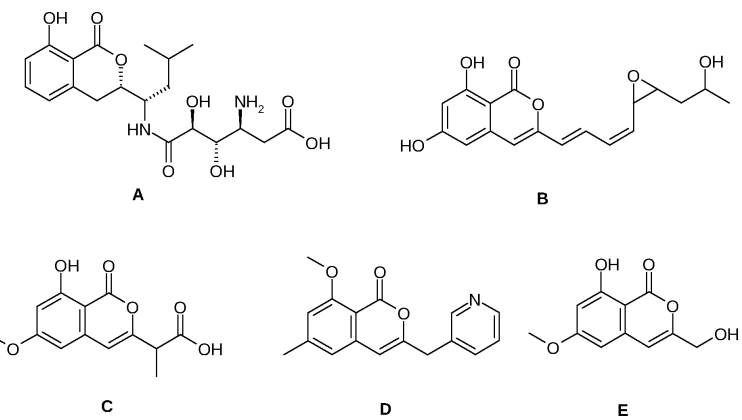

Fig. 1. Structures of AI-77-B (A), paraphaeosphaerin A (B), NM-3 (C), 185322 (D), and cytogenin (E).

pounds are oxygen substituents (mainly at C-8 and to a lesser extent at C-6), and side chains at C-3.

Since there is a large structural diversity in these side chains (length, branching, polarity, additional functional groups), we intended to get more insight into the influence of the C-3 substituents on cytotoxic activity. For this purpose a series of isocoumarins and dihydroisocoumarins with different substituents at C-3 was to be synthesized and screened for cytotoxic activity.

In addition to the classical synthetic approaches to the isocoumarin ring system starting from homophthalic acid and related compounds [2,3], organometallic methods have been introduced a few decades ago $[10,11]$. In 1993, Kundu and Pal reported on the coupling of 2-iodobenzoic acid with terminal alkynes catalyzed by $\mathrm{Pd}\left(\mathrm{PPh}_{3}\right)_{2} \mathrm{Cl}_{2}, \mathrm{CuI}$ and $\mathrm{Et}_{3} \mathrm{~N}$ for the prepara- 
<smiles>CC(=O)c1ccccc1I</smiles>

1a: $\mathrm{X}=\mathrm{OH}$ 1b: $\mathrm{X}=\mathrm{Cl}$
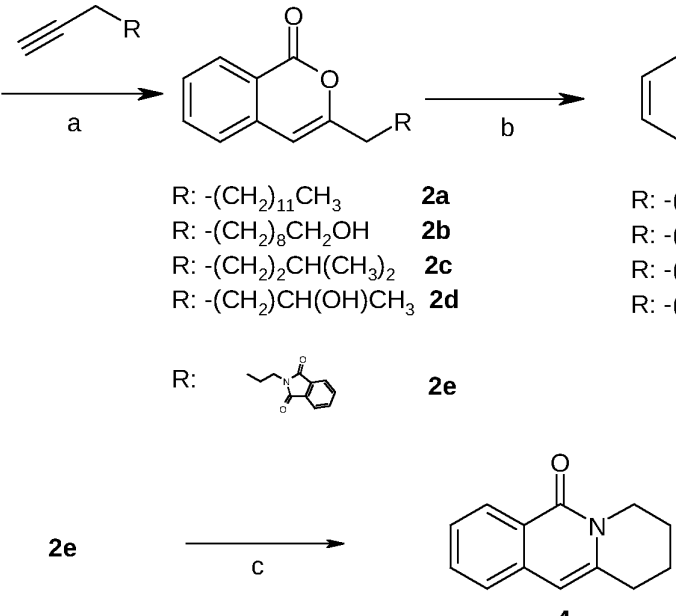

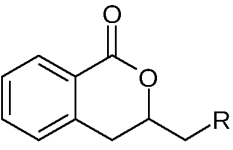

R: $-\left(\mathrm{CH}_{2}\right)_{11} \mathrm{CH}_{3} \quad \mathbf{3 a}$

R: $-\left(\mathrm{CH}_{2}\right)_{8} \mathrm{CH}_{2} \mathrm{OH}: \quad 3 b$

R: $-\left(\mathrm{CH}_{2}\right)_{2} \mathrm{CH}\left(\mathrm{CH}_{3}\right)_{2} \quad 3 \mathrm{c}$

: $-\left(\mathrm{CH}_{2}\right) \mathrm{CH}(\mathrm{OH}) \mathrm{CH}_{3}$ 3d
Scheme 1. a: Triethylamine, $\mathrm{ZnCl}_{2}, \mathrm{Pd}\left(\mathrm{PPh}_{3}\right)_{2} \mathrm{Cl}_{2}, \quad \mathrm{DMF}$, $100{ }^{\circ} \mathrm{C} ; \mathrm{b}: \mathrm{H}_{2}, \mathrm{Pd} / \mathrm{C}$, methanol, room temp; c: hydrazine, EtOH, reflux. tion of phthalides [12]. In these reactions isocoumarins were obtained as minor by-products. Later on Liao and Cheng described an efficient method for the synthesis of isocoumarins from 2-iodobenzoic acid and terminal alkynes using $\mathrm{Pd}\left(\mathrm{PPh}_{3}\right)_{4}$ or $\mathrm{Pd}\left(\mathrm{PPh}_{3}\right)_{2} \mathrm{Cl}_{2}$, $\mathrm{ZnCl}_{2}$ and $\mathrm{Et}_{3} \mathrm{~N}$ as catalysts [13]. We adopted this method for the preparation of our target compounds. In a recent publication it has been shown that the $\mathrm{Pd}$-phosphine complexes can be replaced by $\mathrm{Pd}$ on charcoal [14].

\section{Results}

2-Iodobenzoic acid (1a) was used for Sonogashiratype reactions under catalysis of $\mathrm{Pd}\left(\mathrm{PPh}_{3}\right)_{2} \mathrm{Cl}_{2}$ and $\mathrm{ZnCl}_{2}$ with five terminal alkynes to give directly, due to an intramolecular addition of the carboxylate to the alkyne, the isocoumarins $2 \mathbf{a}-\mathbf{e}$ in 41 to $95 \%$ yield (Scheme 1). In an alternative approach, carboxylic acid 1a was replaced by 2 -iodobenzoyl chloride $\mathbf{1 b}$ and reacted with 5-methylhex-1-yne under otherwise unchanged conditions. Once again the isocoumarin 2c was formed, albeit in lower yield (64\% compared to $99 \%)$.

In a further variant (Scheme 2), inspired by a report of Villemin and Goussu [15] and an accidental formation of an isocoumarin from an alkynyl ben-

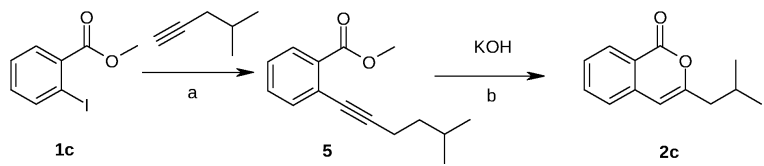

Scheme 2. a: Triethylamine, $\mathrm{CuI}, \mathrm{Pd}\left(\mathrm{PPh}_{3}\right)_{2} \mathrm{Cl}_{2}$, room temp.; b: methanol, reflux. zoic ester in our hands [16], we prepared the alkyne 5 by Sonogashira reaction of methyl 2-iodobenzoate (1c) with 5-methylhex-1-yne under catalysis of $\mathrm{CuI}$ and $\mathrm{Pd}\left(\mathrm{PPh}_{3}\right)_{2} \mathrm{Cl}_{2}$. Upon treatment with methanolic $\mathrm{KOH}$ the isocoumarin 2c was obtained in very low yield $(6 \%)$. Thus the first variant starting from the carboxylic acid 1a was found to give the highest yield, and was exclusively used for the syntheses of the other isocoumarins.

The isocoumarins $\mathbf{2 a}-\mathbf{d}$ were hydrogenated under $\mathrm{Pd} / \mathrm{C}$ catalysis to give the dihydroisocoumarins $\mathbf{3 a}-\mathbf{d}$ (Scheme 1) [17].

An attempt to liberate the primary amino group protected as the $N$-alkylphthalimide in $\mathbf{2 e}$ by hydrazinolysis did not give the expected aminobutyl isocoumarin, but led to the known 1,2,3,4-tetrahydro-pyrido[1,2- $b$ ] isoquinolin-6-one (4) (Scheme 1) [18, 19].

The antibacterial and antimycotic activities of the new compounds were determined in an agar diffusion assay against the bacteria Escherichia coli, Staphylococcus equorum, Pseudomonas antimicrobia, and Streptococcus entericus, and the fungi Aspergillus niger, Candida glabrata, Hypopichii burtonii, and Yarrowia lipolytica. The compounds did not show significant activities compared to the references tetracycline and clotrimazol (data not shown) [20].

Finally, the cytotoxicity of the compounds was determined in a MTT assay on a human leukaemia cell line (HL 60) with cisplatin as a reference [21]. The results are shown in Table 1.

Seven of these compounds showed moderate cytotoxicity with $\mathrm{IC}_{50}$ values in the range of $40-80 \mu \mathrm{M}$. We could detect no significant differences in the cy- 
Table 1. MTT assay: $\mathrm{IC}_{50}$ values against HL-60 cells.

\begin{tabular}{llll}
\hline Compound & $\mathrm{IC}_{50}(\mu \mathrm{M})$ & Compound & $\mathrm{IC}_{50}(\mu \mathrm{M})$ \\
\hline $\mathbf{2 a}$ & 45 & $\mathbf{3 a}$ & 44 \\
$\mathbf{2 b}$ & 70 & $\mathbf{3 b}$ & 62 \\
$\mathbf{2 c}$ & 40 & $\mathbf{3 c}$ & 78 \\
$\mathbf{2 d}$ & $>100(3547)$ & $\mathbf{3 d}$ & $>100(1978)$ \\
$\mathbf{2 e}$ & 48 & $\mathbf{4}$ & $>100(354)$ \\
cisplatin & 5 & & \\
\hline
\end{tabular}

Table 2. Values of $\log P$ of compounds $2 \mathbf{a}-\mathbf{e}, \mathbf{3 a}-\mathbf{d}$ and 4 .

\begin{tabular}{llll}
\hline Compound & $\log P$ & Compound & $\log P$ \\
\hline $\mathbf{2 a}$ & 6.5 & 3a & 6.1 \\
2b & 4.3 & 3b & 3.9 \\
2c & 4.3 & 3c & 3.6 \\
2d & 2.0 & 3d & 1.7 \\
2e & 3.6 & $\mathbf{4}$ & 3.3 \\
\hline
\end{tabular}

totoxic potencies of the isocoumarins and the corresponding dihydroisocoumarins.

The extremely poor activities of isocoumarin $\mathbf{2 d}$ and dihydroisocoumarin 3d might be explained by the correlation between cytotoxicity and $\log P$ values. The $\log P$ values of the compounds were determined by a HPLC method by comparison with known $\log P$ values of diphenyl ether, cinnamic acid, aniline and naphthaline [22], and are shown in Table 2. The inactive compounds $\mathbf{2 d}$ and $\mathbf{3 d}$ have very low $\log P$ values.

\section{Discussion}

Five new 3-substituted isocoumarins and four new dihydroisocoumarins were prepared in a convenient one-pot synthesis, and most of them showed very moderate cytotoxic activities, with the isocoumarins and the corresponding dihydro derivatives exhibiting almost equal potency. The differences in cytotoxic activity might be explained on the basis of $\log P$ values. Since none of the compounds prepared here has cytotoxic activity comparable to the drug candidates NM-3 and 185322, substitution (hydroxy and methoxy groups) at the benzoid ring of the isocoumarins seems to be more crucial than the nature of the substituents at C-3.

\section{Experimental Section}

Elemental analysis: Heraeus CHN Rapid; IR spectra: Perkin-Elmer FT-IR Paragon 1000; MS: Hewlett Packard MS-Engine, electron ionization (EI) $70 \mathrm{eV}$, chemical ionization (CI) with $\mathrm{CH}_{4}(300 \mathrm{eV})$; GLC-MS: Shimadzu GC 17 A, EI (70 eV); NMR: Jeol GSX $400\left({ }^{1} \mathrm{H}\right.$ : $400 \mathrm{MHz}$, ${ }^{13} \mathrm{C}$ : $100 \mathrm{MHz}$; melting points were determined on a
Büchi melting point B-540 apparatus and are uncorrected; flash column chromatography (FCC): silica gel 60 (230400 mesh, E. Merck, Darmstadt); HPLC: Merck Hitachi Series 7000, column: LiChroCart ${ }^{\circledR}$ 250-4; LiChrospher 100 RP 18 ( $5 \mu \mathrm{M}$, Merck), eluent: methanol/ water $75: 25$ (compound 2a and 3a: $90: 10$ ), detection: $\mathrm{UV}, \lambda=254 \mathrm{~nm}$.

\section{General procedure 1 (Sonogashira reaction)}

$\mathrm{Pd}\left(\mathrm{PPh}_{3}\right)_{2} \mathrm{Cl}_{2}(0.10 \mathrm{mmol})$ and $\mathrm{ZnCl}_{2}(4.0 \mathrm{mmol})$ were added under $\mathrm{N}_{2}$ atmosphere to a solution of aryl iodide $(2.00 \mathrm{mmol})$, alkyne $(6.0 \mathrm{mmol})$, and triethylamine $(10 \mathrm{mmol})$ in DMF $(2 \mathrm{~mL})$. The suspension was heated at $100{ }^{\circ} \mathrm{C}$ for $24 \mathrm{~h}$. The components of the suspension were separated by flash column chromatography ( $n$-hexane / ethyl acetate).

\section{General procedure 2 (catalytic hydrogenation)}

$20 \mathrm{mg}$ of Pd on charcoal (10\%) was added to a solution of the isocoumarin in methanol $(20 \mathrm{~mL})$. The suspension was stirred for $12 \mathrm{~h}$ under $\mathrm{H}_{2}$ atmosphere, then the catalyst was filtered off, and the residue was washed with methanol. The combined organic layers were evaporated, and the residue was purified by FCC ( $n$-hexane / ethyl acetate).

\section{3-Dodecylisochromen-1-one (2a)}

Compound 2a was prepared following general procedure 1 from 2-iodobenzoic acid (496 mg, $2.00 \mathrm{mmol}$ ), tetradec1-yne $(1.17 \mathrm{~g}, 6.00 \mathrm{mmol})$, triethylamine $(1.0 \mathrm{~g}, 10 \mathrm{mmol})$, $\mathrm{Pd}\left(\mathrm{PPh}_{3}\right)_{2} \mathrm{Cl}_{2}(70 \mathrm{mg}, 0.10 \mathrm{mmol})$, and $\mathrm{ZnCl}_{2}(545 \mathrm{mg}$, $4.00 \mathrm{mmol}$ ) to give $560 \mathrm{mg}(89 \%)$ of $\mathbf{2 a}$ as a brown solid. M. p.: $48{ }^{\circ} \mathrm{C} .-\mathrm{C}_{21} \mathrm{H}_{30} \mathrm{O}_{2}$ (314.47): calcd. C 80.21, H 9.62; found $\mathrm{C} 80.12, \mathrm{H} 9.95 .-{ }^{1} \mathrm{H} \mathrm{NMR}\left(\mathrm{CDCl}_{3}\right): \delta=0.88(\mathrm{t}, J=$ $\left.7.1 \mathrm{~Hz}, 3 \mathrm{H}, \mathrm{CH}_{3}\right), 1.27\left(\mathrm{~m}, 18 \mathrm{H}, 9 \mathrm{CH}_{2}, 3^{\prime} \mathrm{H}-11^{\prime} \mathrm{H}\right), 1.72$ (tt, $\left.J_{1}=J_{2}=7.4 \mathrm{~Hz}, 2 \mathrm{H}, \mathrm{CH}_{2}, 2^{\prime}-\mathrm{H}\right), 2.53(\mathrm{t}, J=7.4 \mathrm{~Hz}$, $\left.\mathrm{CH}_{2}, 2 \mathrm{H}, 1^{\prime}-\mathrm{H}\right), 6.28$ (s, $1 \mathrm{H}$, arom. $\left.\mathrm{CH}, 4-\mathrm{H}\right), 7.37$ (d, $J=$ $7.8 \mathrm{~Hz}, 1 \mathrm{H}$, arom. CH, 6-H), $7.46\left(\mathrm{ddd}, J_{1}=J_{2}=7.8 \mathrm{~Hz}\right.$, $J_{3}=1.4 \mathrm{~Hz}, 1 \mathrm{H}$, arom. CH, 8-H), $7.68\left(\mathrm{ddd}, J_{1}=J_{2}=7.8 \mathrm{~Hz}\right.$, $J_{3}=1.4 \mathrm{~Hz}, 1 \mathrm{H}$, arom. CH, 7-H), $8.27(\mathrm{~d}, J=7.8 \mathrm{~Hz}, 1 \mathrm{H}$, arom. $\mathrm{CH}, 9-\mathrm{H}) .-{ }^{13} \mathrm{C} \mathrm{NMR}\left(\mathrm{CDCl}_{3}\right): \delta=14.12\left(\mathrm{CH}_{3}\right)$, $22.70\left(\mathrm{C}-11^{\prime}\right), 26.93\left(\mathrm{C}-2^{\prime}\right), 29.02\left(\mathrm{CH}_{2}\right), 29.04\left(\mathrm{CH}_{2}\right)$, $29.33\left(\mathrm{CH}_{2}\right), 29.50\left(\mathrm{CH}_{2}\right), 29.51\left(\mathrm{CH}_{2}\right), 29.62\left(\mathrm{CH}_{2}\right), 29.63$ $\left(\mathrm{CH}_{2}\right), 31.93\left(\mathrm{CH}_{2}\right), 33.56\left(\mathrm{C}-1^{\prime}\right), 102.85(\mathrm{C}-4), 120.17$ (C-10), 125.01 (C-6), 127.52 (C-8), 129.53 (C-9), 134.69 (C-7), 137.68 (C-5), 158.40 (C-3), 163.12 (CO). - IR (KBr): $v=2924,2853,1732,1657,1607,1569,1484,1466,1363$, 1326, 1289, 1240, 1204, 1160, 1110, 1046, 1021, 968, 823, 756, $690 \mathrm{~cm}^{-1}$. - MS (EI): $m / z(\%)=314(38)[\mathrm{M}]^{+}, 173$ (28), 160 (61), 118 (100). - MS (CI): $\mathrm{m} / z(\%)=315$ (100) $[\mathrm{M}+1]^{+}$.

\section{3-(9-Hydroxynonyl)-isochromen-1-one (2b)}

The compound was prepared following general procedure 1 from 2-iodobenzoic acid (496 mg, $2.00 \mathrm{mmol})$, undec-10- 
yn-1-ol $(1.0 \mathrm{~g}, 6.0 \mathrm{mmol})$, triethylamine $(1.0 \mathrm{~g}, 10 \mathrm{mmol})$, $\mathrm{Pd}\left(\mathrm{PPh}_{3}\right)_{2} \mathrm{Cl}_{2}(70 \mathrm{mg}, 0.10 \mathrm{mmol})$, and $\mathrm{ZnCl}_{2}(545 \mathrm{mg}$, $4.00 \mathrm{mmol})$ to give $443 \mathrm{mg}(77 \%)$ of $\mathbf{2 b}$ as a pale-yellow solid. - M. p.: $63{ }^{\circ}$ C. $-\mathrm{C}_{18} \mathrm{H}_{24} \mathrm{O}_{3}(288.39)$ : calcd. C 74.97, $\mathrm{H}$ 8.39; found $\mathrm{C} 74.62, \mathrm{H} 8.48 .-{ }^{1} \mathrm{H}$ NMR $\left(\mathrm{CDCl}_{3}\right): \delta=$ $1.34\left(\mathrm{~m}, 10 \mathrm{H}, 5 \mathrm{CH}_{2}, 3^{\prime}-\mathrm{H}-7^{\prime}-\mathrm{H}\right), 1.57\left(\mathrm{tt}, J_{1}=J_{2}=6.7 \mathrm{~Hz}\right.$, $\left.2 \mathrm{H}, \mathrm{CH}_{2}, 8^{\prime}-\mathrm{H}\right), 1.72\left(\mathrm{tt}, J_{1}=J_{2}=7.7 \mathrm{~Hz}, 2 \mathrm{H}, \mathrm{CH}_{2}, 2^{\prime}-\mathrm{H}\right)$, $2.53\left(\mathrm{t}, J=7.7 \mathrm{~Hz}, 2 \mathrm{H}, \mathrm{CH}_{2}, 1^{\prime}-\mathrm{H}\right), 3.65(\mathrm{t}, J=6.7 \mathrm{~Hz}$, $\left.2 \mathrm{H}, \mathrm{CH}_{2}, 9^{\prime}-\mathrm{H}\right), 6.27$ (s, $1 \mathrm{H}$, arom. $\left.\mathrm{CH}, 4-\mathrm{H}\right), 7.37$ (d, $J=$ $7.8 \mathrm{~Hz}, 1 \mathrm{H}$, arom. CH, 6-H), $7.46\left(\mathrm{ddd}, J_{1}=J_{2}=7.8 \mathrm{~Hz}\right.$, $J_{3}=1.3 \mathrm{~Hz}, 1 \mathrm{H}$, arom. CH, 8-H), $7.68\left(\mathrm{ddd}, J_{1}=J_{2}=\right.$ $7.8 \mathrm{~Hz}, J_{3}=1.3 \mathrm{~Hz}, 1 \mathrm{H}$, arom. $\left.\mathrm{CH}, 7-\mathrm{H}\right), 8.26(\mathrm{~d}, J=$ $7.8 \mathrm{~Hz}, 1 \mathrm{H}$, arom. $\mathrm{CH}, 9-\mathrm{H}) .-{ }^{13} \mathrm{C} \mathrm{NMR}\left(\mathrm{CDCl}_{3}\right): \delta=$ $25.82\left(\mathrm{CH}_{2}\right), 27.01\left(\mathrm{C}-2^{\prime}\right), 29.09\left(\mathrm{CH}_{2}\right), 29.33\left(\mathrm{CH}_{2}\right), 29.46$ $\left(\mathrm{CH}_{2}\right), 29.52\left(\mathrm{CH}_{2}\right), 32.88\left(\mathrm{C}-8^{\prime}\right), 33.67\left(\mathrm{C}-1^{\prime}\right), 63.04\left(\mathrm{C}-9^{\prime}\right)$, 102.90 (C-4), 120.14 (C-10), 125.02 (C-6), 127.55 (C-8), 129.52 (C-9), 134.72 (C-7), 137.66 (C-5), 158.32 (C-3), 163.16 (CO). - IR (KBr): $v=3437,2927,2854,1730,1655$, 1483, 1277, 1161, 1055, 1022, 756, $692 \mathrm{~cm}^{-1}$. - MS (EI): $\mathrm{m} / \mathrm{z}(\%)=288(100)[\mathrm{M}]^{+}, 270(15), 258(30) .-\mathrm{MS}(\mathrm{CI}):$ $m / z(\%)=289(100)[\mathrm{M}+1]^{+} .-$HR-MS (EI): $m / z=288.1752$ (calcd. 288.1726 for $\mathrm{C}_{18} \mathrm{H}_{24} \mathrm{O}_{3},[\mathrm{M}]^{+}$).

\section{3-(3-Methylbutyl)isochromen-1-one (2c)}

a) The compound was prepared following general procedure 1 from 2-iodobenzoic acid (496 mg, $2.00 \mathrm{mmol})$, 5methylhex-1-yne $(577 \mathrm{mg}, 6.00 \mathrm{mmol})$, triethylamine $(1.0 \mathrm{~g}$, $10 \mathrm{mmol}), \mathrm{Pd}\left(\mathrm{PPh}_{3}\right)_{2} \mathrm{Cl}_{2}(70 \mathrm{mg}, 0.10 \mathrm{mmol})$ and $\mathrm{ZnCl}_{2}$ (545 $\mathrm{mg}, 4.00 \mathrm{mmol})$ to give $410 \mathrm{mg}(95 \%)$ of $2 \mathrm{c}$ as a brown oil.

b) Alternatively the compound was prepared following general procedure 1 from 2-iodobenzoyl chloride $(533 \mathrm{mg}$, $2.00 \mathrm{mmol})$, 5-methylhex-1-yne (577 mg, $6.00 \mathrm{mmol})$, triethylamine $(1.0 \mathrm{~g}, 10 \mathrm{mmol}), \mathrm{Pd}\left(\mathrm{PPh}_{3}\right)_{2} \mathrm{Cl}_{2}(70 \mathrm{mg}$, $0.10 \mathrm{mmol}$ ), and $\mathrm{ZnCl}_{2}$ (545 mg, $\left.4.00 \mathrm{mmol}\right)$ to give $276 \mathrm{mg}$ $(64 \%)$ of $2 \mathrm{c}$ as a brown oil.

c) $700 \mathrm{mg}$ (3.04 mmol) of $\mathbf{5}$ was dissolved in $50 \mathrm{~mL}$ of a methanolic $\mathrm{KOH}$ solution $(5 \%)$ and refluxed for $48{ }^{\circ} \mathrm{C}$. The solution was neutralized with hydrochloric acid, diluted with water and extracted with diethyl ether $(3 \times 40 \mathrm{~mL})$. The combined organic layers were dried over $\mathrm{Na}_{2} \mathrm{SO}_{4}$, the solvent was evaporated, and the residue was purified by flash column chromatography (ethyl acetate/ $n$-hexane $1: 1$ ) to give $40 \mathrm{mg}(6 \%)$ of $2 \mathrm{c}$ as a brown oil. $-\mathrm{C}_{14} \mathrm{H}_{16} \mathrm{O}_{2}$ (216.28): calcd. C 77.75, $\mathrm{H}$ 7.46; found $\mathrm{C} 77.89$, $\mathrm{H}$ 8.02. - ${ }^{1} \mathrm{H}$ NMR $\left(\mathrm{CDCl}_{3}\right): \delta=0.95\left(\mathrm{dt}, J_{1}=6.2 \mathrm{~Hz}, J_{2}=1.8 \mathrm{~Hz}, 6 \mathrm{H}\right.$, $2 \mathrm{CH}_{3}, 1^{\prime \prime}-\mathrm{H}$ and $\left.4^{\prime}-\mathrm{H}\right), 1.62\left(\mathrm{~m}, 3 \mathrm{H}, 2^{\prime}-\mathrm{H}\right.$ and $\left.3^{\prime} \mathrm{H}\right), 2.54$ (t, $\left.J=7.2 \mathrm{~Hz}, 2 \mathrm{H}, \mathrm{CH}_{2} 1^{\prime}-\mathrm{H}\right), 6.26(\mathrm{~s}, 1 \mathrm{H}$, arom. $\mathrm{CH}$, 4-H), 7.36 (d, $J=7.8 \mathrm{~Hz}, 1 \mathrm{H}$, arom. CH, 6-H), 7.44 (dd, $J_{1}=J_{2}=7.8 \mathrm{~Hz}, 1 \mathrm{H}$, arom. CH, 8-H), $7.69\left(\mathrm{dd}, J_{1}=J_{2}=\right.$ $7.8 \mathrm{~Hz}, 1 \mathrm{H}$, arom. CH, 7-H), $8.27(\mathrm{~d}, J=7.8 \mathrm{~Hz}, 1 \mathrm{H}$, arom. $\mathrm{CH}, 9-\mathrm{H}) .-{ }^{13} \mathrm{C} \mathrm{NMR}\left(\mathrm{CDCl}_{3}\right): \delta=22.35\left(\mathrm{C}-1^{\prime \prime}\right.$ and C-4'), $27.59\left(\mathrm{C}-3^{\prime}\right), 31.52\left(\mathrm{C}-2^{\prime}\right), 35.87\left(\mathrm{C}-1^{\prime}\right), 102.74(\mathrm{C}-4)$,
120.10 (C-10), 125.01 (C-6), 127.52 (C-8), 129.49 (C-9), 134.71 (C-7), 137.67 (C-5), 158.56 (C-3), 163.13 (CO). - IR (NaCl, film): $v=2956,2928,2870,1732,1657,1606,1569$, 1484, 1468, 1367, 1328, 1161, 1108, 1048, 1022, 968, 823, $757,691 \mathrm{~cm}^{-1}$. - MS (EI): $\mathrm{m} / z(\%)=216(12)[\mathrm{M}]^{+}, 160$ (25), $118(100)$. - MS (CI): $m / z(\%)=217(100)[\mathrm{M}+1]^{+}$.

\section{( \pm )-3-(2-Hydroxypropyl)-isochromen-1-one (2d)}

The compound was prepared following general procedure 1 from 2-iodobenzoic acid (496 mg, $2.00 \mathrm{mmol}$ ), pent-4-yn2-ol (505 mg, $6.00 \mathrm{mmol})$, triethylamine $(1.0 \mathrm{~g}, 10 \mathrm{mmol})$, $\mathrm{Pd}\left(\mathrm{PPh}_{3}\right)_{2} \mathrm{Cl}_{2}$ (70 mg, $\left.0.10 \mathrm{mmol}\right)$, and $\mathrm{ZnCl}_{2}$ (545 mg, $4.00 \mathrm{mmol}$ ) to give $213 \mathrm{mg}(52 \%)$ of $\mathbf{2 d}$ as a pale yellow oil. $-\mathrm{C}_{12} \mathrm{H}_{12} \mathrm{O}_{3}$ (204.23): calcd. C 70.58, H 5.92; found C 69.81, H 6.21. $-{ }^{1} \mathrm{H}$ NMR $\left(\mathrm{CDCl}_{3}\right): \delta=1.33(\mathrm{~d}, J=$ $\left.6.3 \mathrm{~Hz}, 3 \mathrm{H}, \mathrm{CH}_{3}\right), 2.64\left(\mathrm{dd}, J_{1}=14.4 \mathrm{~Hz}, J_{2}=8.1 \mathrm{~Hz}\right.$, $\left.1 \mathrm{H}, 1^{\prime}-\mathrm{H}\right), 2.71\left(\mathrm{dd}, J_{1}=14.4 \mathrm{~Hz}, J_{2}=4.4 \mathrm{~Hz}, 1 \mathrm{H}, 1^{\prime}-\mathrm{H}\right)$, 4.32 (m, $\left.1 \mathrm{H}, \mathrm{CH}, 2^{\prime}-\mathrm{H}\right), 6.39$ (s, $1 \mathrm{H}$, arom. $\left.\mathrm{CH}, 4-\mathrm{H}\right), 7.39$ $\left(\mathrm{d}, J=7.8 \mathrm{~Hz}, 1 \mathrm{H}\right.$, arom. CH, 6-H), 7.48 (ddd, $J_{1}=J_{2}=$ $7.8 \mathrm{~Hz}, J_{3}=1.4 \mathrm{~Hz}, 1 \mathrm{H}$, arom. $\left.\mathrm{CH}, 8-\mathrm{H}\right), 7.70\left(\mathrm{ddd}, J_{1}=\right.$ $J_{2}=7.8 \mathrm{~Hz}, J_{3}=1.4 \mathrm{~Hz}, 1 \mathrm{H}$, arom. $\left.\mathrm{CH}, 7-\mathrm{H}\right), 8.24(\mathrm{~d}$, $J=7.8 \mathrm{~Hz}, 1 \mathrm{H}$, arom. $\mathrm{CH}, 9-\mathrm{H}) .-{ }^{13} \mathrm{C} \mathrm{NMR}\left(\mathrm{CDCl}_{3}\right)$ : $\delta=23.28\left(\mathrm{CH}_{3}\right), 43.28\left(\mathrm{C}-1^{\prime}\right), 65.59\left(\mathrm{C}-2^{\prime}\right), 105.09(\mathrm{C}-4)$, 120.25 (C-10), 125.24 (C-6), 127.94 (C-8), 129.55 (C-9), 134.85 (C-7), 137.28 (C-5), 154.96 (C-3), 162.84 (CO). IR (NaCl, film): $v=3429,2970,2918,1718,1655,1606$, 1483, 1325, 1163, 1119, 1045, 1024, 972, 937, 823, 758, $690 \mathrm{~cm}^{-1}$. - MS (EI): $\mathrm{m} / z(\%)=204(16)[\mathrm{M}]^{+}, 160(100)$, $131(25) .-\mathrm{MS}(\mathrm{CI}): \mathrm{m} / z(\%)=205(100)[\mathrm{M}+1]^{+}$. - HR-MS: $m / z=204.0777$ (calcd. 204.0787 for $\mathrm{C}_{12} \mathrm{H}_{12} \mathrm{O}_{3},[\mathrm{M}]^{+}$).

\section{2-[4-(1-Oxo-1H-isochromen-3-yl)-butyl]isoindol-1,3-dione (2e)}

The compound was prepared following general procedure 1 from 2-iodobenzoic acid $(496 \mathrm{mg}, 2.00 \mathrm{mmol}$ ), $N$-(hex-5ynyl)-phthalimide $(1.36 \mathrm{~g}, 6.00 \mathrm{mmol})$, triethylamine $(1.0 \mathrm{~g}$, $10 \mathrm{mmol}), \mathrm{Pd}\left(\mathrm{PPh}_{3}\right)_{2} \mathrm{Cl}_{2}(70 \mathrm{mg}, 0.100 \mathrm{mmol})$, and $\mathrm{ZnCl}_{2}$ (545 mg, $4.00 \mathrm{mmol}$ ) to give $285 \mathrm{mg}(41 \%)$ of $2 \mathrm{e}$ as a white powder. - M.p.: $168{ }^{\circ} \mathrm{C}$. $-\mathrm{C}_{21} \mathrm{H}_{17} \mathrm{NO}_{4}$ (347.37): calcd. C 72.61, H 4.93, N 4.03; found C 72.36, H 5.37, N 3.88. ${ }^{1} \mathrm{H} \mathrm{NMR}\left(\mathrm{CDCl}_{3}\right): \delta=1.78\left(\mathrm{~m}, 4 \mathrm{H}, 2 \mathrm{CH}_{2}, 2^{\prime}-\mathrm{H}\right.$ and $\left.3^{\prime}-\mathrm{H}\right)$, $2.59\left(\mathrm{t}, J=6.7 \mathrm{~Hz}, 2 \mathrm{H}, \mathrm{CH}_{2}, 4^{\prime}-\mathrm{H}\right), 3.74(\mathrm{t}, J=6.5 \mathrm{~Hz}$, $\left.2 \mathrm{H}, \mathrm{CH}_{2}, 1^{\prime}-\mathrm{H}\right), 6.27$ (s, $1 \mathrm{H}$, arom. $\left.\mathrm{CH}, 4^{\prime \prime}-\mathrm{H}\right), 7.35$ (d, $J=$ $8.0 \mathrm{~Hz}, 1 \mathrm{H}$, arom. $\left.\mathrm{CH}, 6^{\prime \prime}-\mathrm{H}\right), 7.45$ (ddd, $J_{1}=J_{2}=8.0 \mathrm{~Hz}$, $J_{3}=0.8 \mathrm{~Hz}, 1 \mathrm{H}$, arom. $\left.\mathrm{CH}, 8^{\prime \prime}-\mathrm{H}\right), 7.67$ (ddd, $J_{1}=J_{2}=$ $8.0 \mathrm{~Hz}, J_{3}=0.8 \mathrm{~Hz}, 1 \mathrm{H}$, arom. CH, $\left.7^{\prime \prime}-\mathrm{H}\right), 7.71\left(\mathrm{dd}, J_{1}=\right.$ $5.4 \mathrm{~Hz}, J_{2}=3.0 \mathrm{~Hz}, 2 \mathrm{H}$, arom. $\mathrm{CH}, 6-\mathrm{H}$ and $\left.7-\mathrm{H}\right), 7.84(\mathrm{dd}$, $J_{1}=5.4 \mathrm{~Hz}, J_{2}=3.0 \mathrm{~Hz}, 2 \mathrm{H}$, arom. CH, 5-H and 8-H), $8.24\left(\mathrm{dd}, J_{1}=8.0 \mathrm{~Hz}, J_{2}=0.8 \mathrm{~Hz}, 1 \mathrm{H}\right.$, arom. $\left.\mathrm{CH}, 9^{\prime \prime}-\mathrm{H}\right)$. ${ }^{13} \mathrm{C}$ NMR $\left(\mathrm{CDCl}_{3}\right): \delta=24.17\left(\mathrm{CH}_{2}\right), 27.90\left(\mathrm{CH}_{2}\right), 32.99$ $\left(\mathrm{C}-4^{\prime}\right), 37.44\left(\mathrm{C}-1^{\prime}\right), 103.29\left(\mathrm{C}-4^{\prime \prime}\right), 120.17\left(\mathrm{C}-10^{\prime \prime}\right), 123.25$ (C-5 and C-8), $125.10\left(\mathrm{C}-6^{\prime \prime}\right), 127.68\left(\mathrm{C}-8^{\prime \prime}\right), 129.52\left(\mathrm{C}-9^{\prime \prime}\right)$, 
132.07 (C-4 and C-9), 133.96 (C-6 and C-7), $134.73\left(\mathrm{C}-7^{\prime \prime}\right)$, $137.45\left(\mathrm{C}-5^{\prime \prime}\right), 157.32\left(\mathrm{C}-3^{\prime \prime}\right), 162.95\left(\mathrm{C}-1^{\prime \prime}\right), 168.41(\mathrm{C}-1$ and $\mathrm{C}-3) .-\mathrm{IR}(\mathrm{KBr}): v=2927,2858,1766,1709,1657$, 1604, 1566, 1506, 1483, 1466, 1435, 1398, 1371, 1338, $1219,1203,1051,914,822,760,721,687 \mathrm{~cm}^{-1} .-\mathrm{MS}(\mathrm{EI})$ : $m / z(\%)=347(74)[\mathrm{M}]^{+}, 187(30), 173(100), 160(54) .-$ MS (CI): $m / z(\%)=348(100)[\mathrm{M}+1]^{+} .-$HR-MS: $m / z=$ 347.1151 (calcd. 347.1158 for $\mathrm{C}_{21} \mathrm{H}_{17} \mathrm{NO}_{4},[\mathrm{M}]^{+}$).

\section{( \pm -3-Dodecylisochroman-1-one (3a)}

The compound was prepared following general procedure 2 from 2a (205 mg, $0.652 \mathrm{mmol})$ to give $110 \mathrm{mg}(53 \%)$ of $\mathbf{3 a}$ as a pale-yellow solid. - M.p.: $49{ }^{\circ} \mathrm{C}$. $-\mathrm{C}_{21} \mathrm{H}_{32} \mathrm{O}_{2}$ (316.49): calcd. C 79.70, H 10.19; found C 79.48, H 10.54.${ }^{1} \mathrm{H} \mathrm{NMR}\left(\mathrm{CDCl}_{3}\right): \delta=0.88\left(\mathrm{t}, J=7.2 \mathrm{~Hz}, 3 \mathrm{H}, \mathrm{CH}_{3}\right), 1.26$ (m, $\left.18 \mathrm{H}, 9 \mathrm{CH}_{2}, 3^{\prime}-\mathrm{H}-11^{\prime}-\mathrm{H}\right), 1.46\left(\mathrm{~m}, 1 \mathrm{H}, 2^{\prime}-\mathrm{H}\right), 1.57$ $\left(\mathrm{m}, 1 \mathrm{H}, 2^{\prime}-\mathrm{H}\right), 1.72\left(\mathrm{~m}, 1 \mathrm{H}, 1^{\prime}-\mathrm{H}\right), 1.88\left(\mathrm{~m}, 1 \mathrm{H}, 1^{\prime}-\mathrm{H}\right)$, $2.94\left(\mathrm{~m}, 2 \mathrm{H}, \mathrm{CH}_{2}, 4-\mathrm{H}\right), 4.52(\mathrm{~m}, 1 \mathrm{H}, \mathrm{CH}, 3-\mathrm{H}), 7.24$ $\left(\mathrm{d}, J=7.8 \mathrm{~Hz}, 1 \mathrm{H}\right.$, arom. CH, 6-H), $7.38\left(\mathrm{dd}, J_{1}=J_{2}=\right.$ $7.8 \mathrm{~Hz}, 1 \mathrm{H}$, arom. CH, 8-H), $7.53\left(\mathrm{ddd}, J_{1}=J_{2}=7.8 \mathrm{~Hz}\right.$, $J_{3}=1.3 \mathrm{~Hz}, 1 \mathrm{H}$, arom. CH, 7-H), $8.09\left(\mathrm{dd}, J_{1}=7.8 \mathrm{~Hz}\right.$, $J_{2}=1.3 \mathrm{~Hz}, 1 \mathrm{H}$, arom. $\left.\mathrm{CH}, 9-\mathrm{H}\right) .-{ }^{13} \mathrm{C} \mathrm{NMR}\left(\mathrm{CDCl}_{3}\right)$ : $\delta=14.14\left(\mathrm{CH}_{3}\right), 22.70\left(\mathrm{CH}_{2}\right), 24.97\left(\mathrm{C}-2^{\prime}\right), 29.41\left(\mathrm{CH}_{2}\right)$, $29.46\left(\mathrm{CH}_{2}\right), 29.55\left(\mathrm{CH}_{2}\right), 29.62\left(2 \mathrm{CH}_{2}\right), 29.70\left(\mathrm{CH}_{2}\right)$, $29.72\left(\mathrm{CH}_{2}\right), 31.98\left(\mathrm{CH}_{2}\right), 33.27(\mathrm{C}-4), 35.05\left(\mathrm{C}-1^{\prime}\right), 78.84$ (C-3), 125.25 (C-10), 127.34 (C-6), 127.58 (C-8), 130.26 (C-9), 133.62 (C-7), 139.25 (C-5), 165.74 (CO). - IR (KBr): $v=2918,2850,1714,1608,1473,1462,1437,1369,1288$, 1244, 1232, 1119, 1088, 1030, 1001, 741, $694 \mathrm{~cm}^{-1} .-\mathrm{MS}$ (EI): $m / z(\%)=316(22)[\mathrm{M}]^{+}, 147(100), 136(31), 118$ (89). $-\mathrm{MS}(\mathrm{CI}): m / z(\%)=317(100)[\mathrm{M}+1]^{+}$.

\section{( \pm )-3-(9-Hydroxynonyl)-isochroman-1-one (3b)}

The compound was prepared following general procedure 2 from $\mathbf{2 b}(200 \mathrm{mg}, 0.694 \mathrm{mmol})$ to give $200 \mathrm{mg}(99 \%)$ of $\mathbf{3 b}$ as a white solid. - M. p.: $63{ }^{\circ} \mathrm{C} .-\mathrm{C}_{18} \mathrm{H}_{26} \mathrm{O}_{3}$ (290.41): calcd. C 74.45, H 9.02; found C 74.69, H 9.18. - ${ }^{1} \mathrm{H} \mathrm{NMR}\left(\mathrm{CDCl}_{3}\right)$ : $\delta=1.32\left(\mathrm{~m}, 10 \mathrm{H}, 5 \mathrm{CH}_{2}, 3^{\prime}-\mathrm{H}-7^{\prime}-\mathrm{H}\right), 1.57\left(\mathrm{~m}, 4 \mathrm{H}, 2 \mathrm{CH}_{2}\right.$, $2^{\prime}-\mathrm{H}$ and $\left.8^{\prime}-\mathrm{H}\right), 1.72\left(\mathrm{~m}, 1 \mathrm{H}, 1^{\prime}-\mathrm{H}\right), 1.88\left(\mathrm{~m}, 1 \mathrm{H}, 1^{\prime}-\mathrm{H}\right)$, $2.90\left(\mathrm{dd}, J_{1}=16.3 \mathrm{~Hz}, J_{2}=3.5 \mathrm{~Hz}, 1 \mathrm{H}, 4-\mathrm{H}\right), 2.99\left(\mathrm{dd}, J_{1}=\right.$ $\left.16.3 \mathrm{~Hz}, J_{2}=11.0 \mathrm{~Hz}, 1 \mathrm{H}, 4-\mathrm{H}\right), 3.65(\mathrm{t}, J=6.6 \mathrm{~Hz}, 2 \mathrm{H}$, $\left.\mathrm{CH}_{2}, 9^{\prime}-\mathrm{H}\right), 4.52(\mathrm{~m}, 1 \mathrm{H}, \mathrm{CH}, 3-\mathrm{H}), 7.24(\mathrm{~d}, J=7.6 \mathrm{~Hz}, 1 \mathrm{H}$, arom. $\mathrm{CH}, 6-\mathrm{H}), 7.39$ (dd, $J_{1}=J_{2}=7.6 \mathrm{~Hz}, 1 \mathrm{H}$, arom. $\mathrm{CH}$, $8-\mathrm{H}), 7.53$ (ddd, $J_{1}=J_{2}=7.6 \mathrm{~Hz}, J_{3}=1.1 \mathrm{~Hz}, 1 \mathrm{H}$, arom. $\mathrm{CH}, 7-\mathrm{H}), 8.09\left(\mathrm{dd}, J_{1}=7.6 \mathrm{~Hz}, J_{2}=1.1 \mathrm{~Hz}, 1 \mathrm{H}\right.$, arom. CH, 9-H). $-{ }^{13} \mathrm{C} \mathrm{NMR}\left(\mathrm{CDCl}_{3}\right): \delta=24.87\left(\mathrm{C}-2^{\prime}\right), 25.70\left(\mathrm{CH}_{2}\right)$, $29.34\left(\mathrm{CH}_{2}\right), 29.35\left(\mathrm{CH}_{2}\right), 29.38\left(\mathrm{CH}_{2}\right), 29.45\left(\mathrm{CH}_{2}\right), 32.76$ $\left(\mathrm{C}-8^{\prime}\right), 33.39$ (C-4), $35.14\left(\mathrm{C}-1^{\prime}\right), 63.04$ (C-9'), 78.77 (C-3), 125.23 (C-10), 127.35 (C-6), 127.60 (C-8), 130.26 (C-9), 133.64 (C-7), 139.23 (C-5), 165.76 (CO). - IR (KBr): $v=$ 3431, 2925, 2852, 1716, 1608, 1462, 1290, 1122, 1076, 1030, 741, $694 \mathrm{~cm}^{-1}$. - MS (EI): $\mathrm{m} / z(\%)=290(25)[\mathrm{M}]^{+}$,
272 (75), 260 (79), 147 (98), 118 (100). - HR-MS (EI): $\mathrm{m} / \mathrm{z}=$ 290.1894 (calcd. 290.1882 for $\mathrm{C}_{18} \mathrm{H}_{26} \mathrm{O}_{3},[\mathrm{M}]^{+}$).

\section{(土)-3-(3-Methylbutyl)-isochroman-1-one (3c)}

The compound was prepared following general procedure 2 from $2 \mathrm{c}$ (200 mg, $0.925 \mathrm{mmol})$ to give $200 \mathrm{mg}$ (99\%) of 3c as a pale-brown oil. $-\mathrm{C}_{14} \mathrm{H}_{18} \mathrm{O}_{2}$ (218.30): calcd. C 77.03, $\mathrm{H}$ 8.31; found C 77.10, $\mathrm{H} \mathrm{8.76.}{ }^{1} \mathrm{H} \mathrm{NMR}\left(\mathrm{CDCl}_{3}\right): \delta=0.93$ $\left(\mathrm{d}, J=6.8 \mathrm{~Hz}, 6 \mathrm{H}, 2 \mathrm{CH}_{3}\right), 1.34\left(\mathrm{~m}, 1 \mathrm{H}, 2^{\prime}-\mathrm{H}\right), 1.50(\mathrm{~m}$, $\left.1 \mathrm{H}, 2^{\prime}-\mathrm{H}\right), 1.60\left(\mathrm{~m}, 1 \mathrm{H}, \mathrm{CH}, 3^{\prime}-\mathrm{H}\right), 1.75\left(\mathrm{~m}, 1 \mathrm{H}, 1^{\prime}-\mathrm{H}\right)$, $1.88\left(\mathrm{~m}, 1 \mathrm{H}, 1^{\prime}-\mathrm{H}\right), 2.95\left(\mathrm{~m}, 2 \mathrm{H}, \mathrm{CH}_{2}, 4-\mathrm{H}\right), 4.50(\mathrm{~m}$, $1 \mathrm{H}, \mathrm{CH}, 3-\mathrm{H}), 7.24(\mathrm{~d}, J=7.7 \mathrm{~Hz}, 1 \mathrm{H}$, arom. $\mathrm{CH}, 6-\mathrm{H})$, 7.39 (ddd, $J_{1}=J_{2}=7.7 \mathrm{~Hz}, J_{3}=1.0 \mathrm{~Hz}, 1 \mathrm{H}$, arom. $\mathrm{CH}$, 8-H), 7.53 (ddd, $J_{1}=J_{2}=7.7 \mathrm{~Hz}, J_{3}=1.0 \mathrm{~Hz}, 1 \mathrm{H}$, arom. $\mathrm{CH}, 7-\mathrm{H}), 8.09\left(\mathrm{dd}, J_{1}=7.7 \mathrm{~Hz}, J_{2}=1.0 \mathrm{~Hz}, 1 \mathrm{H}\right.$, arom. $\mathrm{CH}, 9-\mathrm{H}) .-{ }^{13} \mathrm{C} \mathrm{NMR}\left(\mathrm{CDCl}_{3}\right): \delta=22.47\left(2 \mathrm{CH}_{3}\right), 27.91$ $\left(\mathrm{C}-3^{\prime}\right), 32.89\left(\mathrm{C}-1^{\prime}\right), 33.24$ (C-4), $33.88\left(\mathrm{C}-2^{\prime}\right), 77.37$ (C-3), 152.21 (C-10), 127.35 (C-6), 127.58 (C-8), 130.24 (C-9), 133.65 (C-7), 139.25 (C-5), 165.75 (CO). - IR (NaCl, film): $v=2954,2870,1726,1608,1460,1385,1367,1281,1252$, $1117,1086,1032,744,694 \mathrm{~cm}^{-1}$. - MS (EI): $\mathrm{m} / \mathrm{z}(\%)=218$ (15) $[\mathrm{M}]^{+}, 162$ (15), 147 (57), 119 (100), 118 (86). - MS (CI): $m / z(\%)=219(100)[\mathrm{M}+1]^{+}-\mathrm{HR}-\mathrm{MS}(\mathrm{EI}): \mathrm{m} / \mathrm{z}=$ 218.1307 (calcd. 218.1309 for $\mathrm{C}_{14} \mathrm{H}_{18} \mathrm{O}_{2},[\mathrm{M}]^{+}$).

\section{3-(2-Hydroxypropyl)-isochroman-1-one (3d)}

The compound was prepared following general procedure 2 from $2 d(55 \mathrm{mg}, 0.759 \mathrm{mmol})$ to give $110 \mathrm{mg}(70 \%)$ of 3d (diastereomeric mixture) as a pale-yellow oil. The diastereomers could not be separated by flash column chromatography. $-{ }^{1} \mathrm{H}$ NMR $\left(\mathrm{CDCl}_{3}\right)$ : diastereomer $1(60 \%)$ : $\delta=1.29\left(\mathrm{~d}, J=6.2 \mathrm{~Hz}, 3 \mathrm{H}, \mathrm{CH}_{3}, 3^{\prime}-\mathrm{H}\right), 1.87(\mathrm{~m}, 1 \mathrm{H}$, $\left.1^{\prime}-\mathrm{H}\right), 2.13\left(\mathrm{~m}, 1 \mathrm{H}, 1^{\prime}-\mathrm{H}\right), 3.00\left(\mathrm{~m}, 2 \mathrm{H}, \mathrm{CH}_{2}, 4-\mathrm{H}\right), 4.19$ $\left(\mathrm{m}, 1 \mathrm{H}, \mathrm{CH}, 2^{\prime}-\mathrm{H}\right), 4.76(\mathrm{~m}, 1 \mathrm{H}, \mathrm{CH}, 3-\mathrm{H}), 7.26(\mathrm{~d}, J=$ $7.7 \mathrm{~Hz}, 1 \mathrm{H}$, arom. CH, 6-H), $7.41\left(\mathrm{dd}, J_{1}=J_{2}=7.7 \mathrm{~Hz}, 1 \mathrm{H}\right.$, arom. $\mathrm{CH}, 8-\mathrm{H}), 7.55\left(\mathrm{dd}, J_{1}=J_{2}=7.7 \mathrm{~Hz}, 1 \mathrm{H}\right.$, arom. $\mathrm{CH}$, 7-H), $8.10(\mathrm{~d}, J=7.7 \mathrm{~Hz}, 1 \mathrm{H}$, arom. $\mathrm{CH}, 9-\mathrm{H})$; diastereomer $2(40 \%)$ : $\delta=1.28\left(\mathrm{~d}, J=6.2 \mathrm{~Hz}, 3 \mathrm{H}, \mathrm{CH}_{3}, 3^{\prime}-\mathrm{H}\right)$, $1.78\left(\mathrm{~m}, 1 \mathrm{H}, 1^{\prime}-\mathrm{H}\right), 2.00\left(\mathrm{~m}, 1 \mathrm{H}, 1^{\prime}-\mathrm{H}\right), 3.00\left(\mathrm{~m}, 2 \mathrm{H}, \mathrm{CH}_{2}\right.$, 4-H), 4.29 (m, 1 H, CH, 2'-H), 4.85 (m, 1 H, CH, 3-H), 7.26 $(\mathrm{d}, J=7.7 \mathrm{~Hz}, 1 \mathrm{H}$, arom. $\mathrm{CH}, 6-\mathrm{H}), 7.41\left(\mathrm{dd}, J_{1}=J_{2}=\right.$ $7.7 \mathrm{~Hz}, 1 \mathrm{H}$, arom. CH, 8-H), $7.55\left(\mathrm{dd}, J_{1}=J_{2}=7.7 \mathrm{~Hz}\right.$, $1 \mathrm{H}$, arom. CH, 7-H), $8.10(\mathrm{~d}, J=7.7 \mathrm{~Hz}, 1 \mathrm{H}$, arom. $\mathrm{CH}$, 9-H). $-{ }^{13} \mathrm{C} \mathrm{NMR}\left(\mathrm{CDCl}_{3}\right)$ : diastereomer 1: $\delta=23.75\left(\mathrm{CH}_{3}\right)$, 33.36 (C-4), $43.73\left({\left.\mathrm{C}-1^{\prime}\right)}^{\prime}, 65.43\left(\mathrm{C}-2^{\prime}\right), 76.01\right.$ (C-3), 125.23 (C-10), 127.40 (C-6), 127.74 (C-8), 130.38 (C-9), 133.84 (C-7), $139.20(\mathrm{C}-5), 165.23(\mathrm{CO})$; diastereomer 2: $\delta=24.26$ $\left(\mathrm{CH}_{3}\right), 33.69(\mathrm{C}-4), 44.00\left(\mathrm{C}-1^{\prime}\right), 63.78\left(\mathrm{C}-2^{\prime}\right), 77.71(\mathrm{C}-3)$, 125.10 (C-10), 127.37 (C-6), 127.67 (C-8), 130.30 (C-9), 133.78 (C-7), 139.40 (C-5), 165.59 (CO). - IR (NaCl, film): $v=3418,2966,2925,1719,1606,1460,1375,1291,1263$, $1117,1086,1031,914,848,800,746,695 \mathrm{~cm}^{-1}$. - MS (CI): 
$m / z(\%)=207(100)[\mathrm{M}+1]^{+} .-\mathrm{HR}-\mathrm{MS}(\mathrm{EI}): \mathrm{m} / z=206.0932$ (calcd. 206.0943 for $\mathrm{C}_{12} \mathrm{H}_{14} \mathrm{O}_{3},[\mathrm{M}]^{+}$).

\section{1,2,3,4-Tetrahydropyrido[1,2-b]isoquinolin-6-one (4)}

$500 \mathrm{mg}(1.44 \mathrm{mmol})$ of $\mathbf{2 e}$ was dissolved in $30 \mathrm{~mL}$ of absolute ethanol, and $2.9 \mathrm{~mL}(2.9 \mathrm{mmol})$ of a $1 \mathrm{M}$ hydrazine solution in THF was added. The mixture was refluxed for $6 \mathrm{~h}$, the suspension was filtered, the filtrate was evaporated, and the residue was purified by flash column chromatography (ethyl acetate / $n$-hexane $3: 1$ ) to give $90 \mathrm{mg}(31 \%)$ of $\mathbf{4}$ as a white solid. The spectroscopic data were in full accordance those described in ref. [19].

\section{Methyl 2-(5-methylhex-1-ynyl)-benzoate (5)}

$\mathrm{CuI}(68 \mathrm{mg}, 0.36 \mathrm{mmol})$ was dissolved in $50 \mathrm{~mL}$ of dry triethylamine, and methyl 2-iodobenzoate (2.06 g, $7.86 \mathrm{mmol})$, $\mathrm{Pd}\left(\mathrm{PPh}_{3}\right)_{2} \mathrm{Cl}_{2}(80 \mathrm{mg}, 0.11 \mathrm{mmol})$ and 5-methylhex-1-yne (756 mg, $7.86 \mathrm{mmol}$ ) were added. The mixture was stirred for $24 \mathrm{~h}$ under $\mathrm{N}_{2}$ atmosphere. The solvent was evaporated, the residue dissolved in $50 \mathrm{~mL}$ of $5 \%$ aqueous $\mathrm{Na}_{2} \mathrm{~S}_{2} \mathrm{O}_{3}$ solution, extracted with diethyl ether $(3 \times 50 \mathrm{~mL})$, and the combined organic layers were dried over $\mathrm{Na}_{2} \mathrm{SO}_{4}$. The sol- vent was evaporated and the residue purified by FCC ( $n$ hexane / ethyl acetate) to give $1.76 \mathrm{~g}(97 \%)$ of $\mathbf{5}$ as a brown oil. $-\mathrm{C}_{15} \mathrm{H}_{18} \mathrm{O}_{2}$ (230.31): calcd. C 78.23, H 7.88; found C 77.91, H 8.42. $-{ }^{1} \mathrm{H} \mathrm{NMR}\left(\mathrm{CDCl}_{3}\right): \delta=0.94(\mathrm{~d}, J=7.1 \mathrm{~Hz}$, $6 \mathrm{H}, 2 \mathrm{CH}_{3}, 1^{\prime \prime}-\mathrm{H}$ and $\left.6-\mathrm{H}\right), 1.55\left(\mathrm{dt}, J_{1}=J_{2}=7.1 \mathrm{~Hz}\right.$, $2 \mathrm{H}, \mathrm{CH}_{2}, 4^{\prime}-\mathrm{H}$ ), 1.79 (tsept, $J_{1}=J_{2}=7.1 \mathrm{~Hz}, 1 \mathrm{H}, \mathrm{CH}$, $\left.5^{\prime}-\mathrm{H}\right), 2.48\left(\mathrm{t}, J=7.1 \mathrm{~Hz}, 2 \mathrm{H}, \mathrm{CH}_{2}, 3^{\prime}-\mathrm{H}\right), 3.91(\mathrm{~s}, 3 \mathrm{H}$, $\mathrm{CH}_{3}, 1^{\prime \prime \prime}-\mathrm{H}$ ), 7.30 (ddd, $J_{1}=1.1 \mathrm{~Hz}, J_{2}=J_{3}=7.8 \mathrm{~Hz}, 1 \mathrm{H}$, arom. $\mathrm{CH}, 5-\mathrm{H}), 7.41\left(\mathrm{ddd}, J_{1}=1.1 \mathrm{~Hz}, J_{2}=J_{3}=7.8 \mathrm{~Hz}\right.$, $1 \mathrm{H}$, arom. CH, 4-H), $7.49(\mathrm{~d}, J=7.8 \mathrm{~Hz}, 1 \mathrm{H}$, arom. $\mathrm{CH}$, 3-H), $7.87(\mathrm{~d}, J=7.8 \mathrm{~Hz}, 1 \mathrm{H}$, arom. $\mathrm{CH}, 6-\mathrm{H}) .-{ }^{13} \mathrm{C} \mathrm{NMR}$ $\left(\mathrm{CDCl}_{3}\right): \delta=17.81\left(\mathrm{C}-3^{\prime}\right), 22.22\left(\mathrm{C}-6^{\prime}\right.$ and $\left.\mathrm{C}-1^{\prime \prime}\right), 27.31$ $\left(\mathrm{CH}, \mathrm{C}-5^{\prime}\right), 37.60\left(\mathrm{C}-4^{\prime}\right), 52.03\left(\mathrm{C}-1^{\prime \prime \prime}\right), 79.40\left(\mathrm{C}-1^{\prime}\right), 96.04$ (C-2'), 124.51 (C-1), 127.11 (C-5), 130.13 (C-6), 131.45 (C-4), 131.98 (C-2), 134.22 (C-3), 167.04 (CO). - IR (NaCl, film): $v=2953,2869,2360,2231,1733,1716,1596,1567$, 1484, 1447, 1432. 1293, 1276, 1249, 1128, 1083, 756, 701, $472 \mathrm{~cm}^{-1}$. - MS (EI): $\mathrm{m} / z(\%)=230(15)[\mathrm{M}]^{+}, 215(32)$, 183 (64), 174 (100), 159 (74), 131 (34), 115 (41). - MS (CI): $\mathrm{m} / \mathrm{z}(\%)=231(100)[\mathrm{M}+1]^{+}$.

\section{Acknowledgement}

We thank Martina Stadler for technical support.
[1] R. A. Hill, in Progress in the Chemistry of Organic Natural Products, Vol. 48, Springer Verlag, Wien, New York, 1986.

[2] E. Napolitano, Org. Prep. Proced. Int. 1997, 29, 631 664.

[3] E. A. Markaryan, Russ. Chem. Rev. 1989, 58, 479493.

[4] Y.-F. Huang, L.-H. Li, L. Tian, H.-M. Hua, Y.-H. Pei, J. Antibiot. 2006, 59, 355-357.

[5] E. M. K. Wijerantne, P. A. Paranagama, A. A. L. Gunatilaka, Tetrahedron 2006, 62, 8439-8446.

[6] C.L. Reimer, N. Agata, J. G. Tamman, M. Bamberger, W. M. Dickerson, G. D. Kamphaus, S. L. Rook, M. Milhollen, R. Fram, R. Kalluri, D. Kufe, S. Kharbanda, Cancer Res. 2002, 62, 789-795.

[7] T. Kawano, N. Agata, S. Kharbanda, D. Avigan, D. Kufe, Cancer Chemother. Pharmacol. 2007, 59, $329-335$.

[8] W. Zhang, K. Krohn, S. Draeger, B. Schulz, J. Nat. Prod. 2008, 71, 1078-1081.

[9] M. Azumi, K.-i. Ogawa, T. Fujita, M. Takeshita, R. Yoshida, T. Furumai, Y. Igarashi, Tetrahedron 2008, 64, 6420-6425.

[10] D. E. Korte, L. S. Hegedus, R. K. Wirth, J. Org. Chem. 1977, 42, 1329 - 1336.

[11] R. C. Larock, S. Varaprath, H. H. Lau, C. A. Fellows, J. Am. Chem. Soc. 1984, 106, 5274-5284.
[12] N. G. Kundu, M. J. Pal, J. Chem. Soc., Chem. Commun. 1993, $86-88$.

[13] H.-Y. Liao, C.-H. Cheng, J. Org. Chem. 1995, 60, $3711-3716$.

[14] V. Subramanian, V. R. Batchu, D. Barange, M. Pal, J. Org. Chem. 2005, 70, 4778-4783.

[15] D. Villemin, D. Goussu, Heterocycles 1989, 29, 1255 1261.

[16] J. Krauss, D. Unterreitmeier, C. Neudert, F. Bracher, Arch. Pharm. Pharm. Med. Chem. 2005, 338, $605-$ 608.

[17] R. A. Hill, R. H. Carter, J. Staunton, J. Chem. Soc., Perkin Trans. 1 1981, 2570-2576.

[18] J. Reisch, H. Labitzke, Arch. Pharm. 1975, 308, $713-$ 716.

[19] M. Menes-Arzate, M. Martinez, R. Cruz-Almanza, J. M. Muchowski, Y.M. Osornio, L.D. Miranda, J. Org. Chem. 2004, 69, 4001-4004.

[20] DIN Deutsches Institut für Normung e. V., Methoden zur Empfindlichkeitsprüfung von mikrobiellen Krankheitserregern gegen Chemotherapeutika, DIN 58940, Teil 3 - Beiblatt 1 und Teil 4 - Beiblatt 1, Beuth Verlag, Berlin 1998.

[21] T. J. Mosmann, Immunol. Methods 1983, 65, 55-59.

[22] S. H. Unger, J. R. Cook, J. S. Hollenberger, J. Pharm. Sci. 1978, 67, $1364-1367$. 